\section{Childhood poverty and abdominal obesity in adulthood: a systematic review}

\author{
Pobreza na infância e obesidade abdominal \\ na vida adulta: revisão sistemática
}

\author{
1 Programa de Pós-graduação \\ em Epidemiologia \\ Universidade Federal de \\ Pelotas, Pelotas, Brasil. \\ 2 Department of Food Science \\ and Nutrition, California \\ Polytechnic State University, \\ San Luis Obispo, U.S.A. \\ Correspondence \\ D. González \\ Programa de Pós-graduação \\ em Epidemiologia, \\ Universidade Federal de \\ Pelotas. \\ Rua Marechal Deodoro 1160, \\ Pelotas, RS \\ 96020-220, Brasil. \\ david.epidemio@gmail.com
}

\begin{abstract}
Adverse socioeconomic conditions in childhood can have lasting effects on health, but evidence is lacking from prospective studies concerning the effects of early poverty on abdominal obesity in adulthood. Cross-sectional studies in adults from middle and high-income countries show that current socioeconomic status is inversely related to obesity in women, but the pattern in men is not consistent. A systematic review was undertaken to assess the influence of early socioeconomic status on waist circumference, hip circumference, and waist-hip ratio in adulthood. Thirteen relevant articles were located (five cross-sectional and eight cohort), including only one from a middle-income country and the remainder from high-income settings. In all the studies, childhood poverty was associated with higher levels of abdominal obesity in women. In men, the associations were weaker, and no clear pattern emerged.
\end{abstract}

Abdominal Fat; Obesity; Poverty
David González 1

Aydin Nazmi 2

Cesar G. Victora ${ }^{1}$

\section{Introduction}

Obesity is an important risk factor for incidence of cardiovascular diseases, diabetes, hypertension, and hypercholesterolemia and related mortality 1 . Prevalence of obesity has increased rapidly in high, medium, and low-income countries 2,3,4,5. Socioeconomic conditions have an important influence on prevalence of obesity, as well as on other risk factors associated with cardiovascular diseases 4,6,7,8.

Obesity in adults has been estimated mainly through body mass index (BMI) 9 . Recent studies show that abdominal obesity, evaluated according to waist-hip ratio (WHR), is capable of predicting incidence of cardiovascular diseases better than other anthropometric measures, including BMI 10,11.

WHR is used as an indicator of the amount of fat tissue deposited in the waist area in relation to the hip structure 10,11. However, increased WHR can result from high waist circumference and/ or reduced hip circumference 12,13 . These two components of WHR can be affected by distinct factors and in different stages of growth 14,15. In addition, waist circumference is directly associated with risk of cardiovascular diseases, while hip circumference shows an inverse relationship 12,14,16, thereby supporting the recommendation that the two components be evaluated separately 13 . 
Since obesity has multifactorial origins, the emerging hypothesis of "early onset of adulthood diseases" has led to increasing interest in understanding the mechanisms by which early exposures might affect nutritional and health status over the course of life. Adverse conditions experienced in the intrauterine period and/or in the early months of life can alter organ functions and predispose to the development of diseases in adulthood due to a process of biological programming 17,18,19,20,21.

The effects of childhood poverty on health and nutritional status in the early years of life have been widely studied 2,22 , but few longitudinal studies have evaluated the consequences for obesity in adulthood 23 . Nearly all studies on the association between socioeconomic status and obesity in adults have a cross-sectional design, in which both variables are measured simultaneously 4,6,8,23. By reviewing the literature, we found 40 studies evaluating the relationship between childhood socioeconomic status and obesity in adulthood, of which only three were conducted in medium or low-income countries: two in Brazil 24,25 and one in China ${ }^{26}$. There are even fewer articles specifically evaluating the association between socioeconomic status and abdominal obesity.

Given the possibility that abdominal obesity is determined early, the current systematic review investigates the association between childhood socioeconomic status and waist circumference, hip circumference, and WHR in adulthood.

\section{Methodology}

The systematic literature review included the MEDLINE, Springerlink, Ovid, LILACS, and SciELO databases. Various combinations of $\mathrm{Med}$ ical Subject Headings (MeSH) descriptors were used for the exposure ("socioeconomic status", "socioeconomic factors, income", "education", "social class", "employment”, "occupational status", "poverty") and outcomes ("waist-hip ratio", “hip", “obesity”, “abdominal tissue”, "adiposity”). Articles published until July 2008 were considered. The search was conducted independently by the two first authors of the present article. The reference lists were compared and the relevant articles selected by title and abstract. All the apparently relevant articles were obtained and reviewed by the first author. The bibliographic references cited by the selected articles were also reviewed, and if relevant they were included in the review.

The review selected all studies with a longitudinal or cross-sectional design that evaluated childhood socioeconomic status as the exposure and waist circumference, hip circumference, and/or WHR when the individuals were at least 18 years of age as the outcomes. The review did not include studies limited to carriers of a specific disease.

The results of different articles were compared, whenever the data allowed, based on the slope index of inequality (SII). The SII represents the linear regression coefficient (beta) with the health indicator as the outcome and an ordinal variable representing socioeconomic status as exposure 27 . This index allows comparing studies that have used different categories of socioeconomic status, since the beta parameter represents the difference between the extremes in social distribution, regardless of the classification used. In the analyses presented below, a value of " 0 " was assigned to poorer individuals and " 1 " to wealthier ones.

The results were interpreted using the conceptual model described in Figure 1. The arrows in the figure indicate the possible causal mechanisms. The most distal determinant is the inheritance of genetic characteristics, which supposedly affects the model's other components, including intra-gestational maternal adiposity, as well as the child's intrauterine and post-natal life $3,23,28$. Genetic load can also influence the parents' socioeconomic status, as in the case of discrimination against black 29 or obese individuals 30 . In the second hierarchical level, parents' socioeconomic status can also affect lifetime adiposity 4,6,7,8,23. This effect is supposedly mediated by the family's nutritional habits and physical activity, the child's disease history (particularly that of infectious diseases), and the individual's own socioeconomic status in adulthood $3,23,28$. This model suggests that controlling for genetic factors, if possible, would be justified by the possibility that such factors are influencing both socioeconomic level and nutritional status. Meanwhile, the results adjusted for behavioral factors (diet, smoking, etc.), childhood nutritional status, and/or current socioeconomic status should be interpreted with caution, due to the possibility that these factors are at least partially influenced by the family's socioeconomic status, thereby constituting mediating factors in the target relationship. 


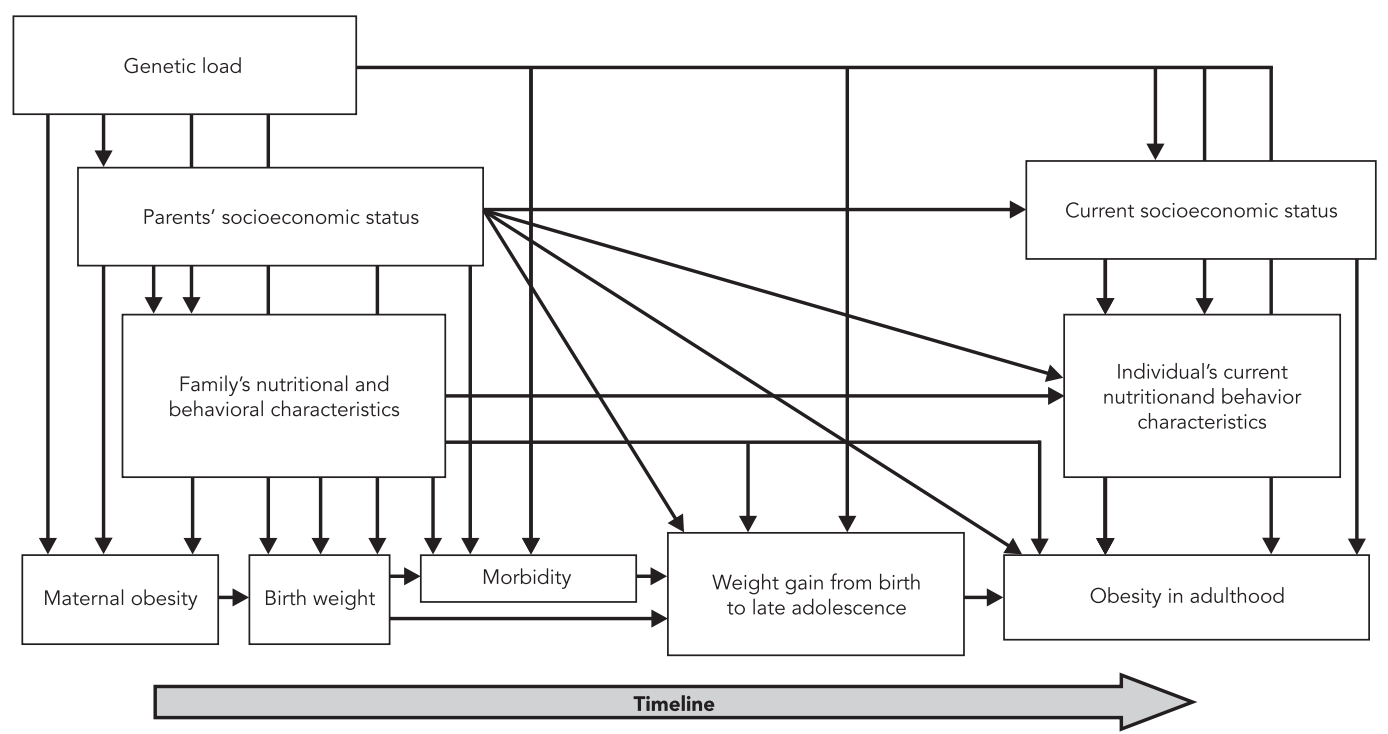

\section{Results}

Figure 2 summarizes the selection process for the reviewed articles, based on the QUOROM guidelines 31 . A total of 1,387 possibly relevant articles were located, of which 134 were selected to be read in full. Ninety-four of these were excluded because they did not evaluate family socioeconomic status when the individual was five years of age or older. Of the remaining articles, only 13 evaluated waist circumference, hip circumference, and/or WHR in adulthood as the outcome $26,32,33,34,35,36,37,38,39,40,41,42,43$.

All the included articles were published in the last ten years. Twelve were performed in highincome countries (11 in Europe 32,33,34,35,36,37,38,39, $40,42,43$ and one in New Zealand 41 ) and one in China 26 . Three British articles 33,36,37 used data from the same cohort; two Finnish articles 34,35 likewise analyzed the same cohort; and two Spanish articles 38,39 used data from the same cross-sectional study, evaluating the outcome differently (continuous or categorical variable) or in distinct moments of life. Thus, the 13 articles refer to nine different studies.

Five articles were based on four studies with a cross-sectional design, which collected information on socioeconomic status retrospectively during interviews with individuals 60 years or older $26,38,39,42,43$. The other articles were based on five studies with prospective longitudinal designs $32,33,34,35,36,37,40,41$. Two Finnish cohorts $32,34,35$ and the New Zealand cohort 41 evaluated young adults (24-39 years), while in the British cohorts $33,36,37,40$ the samples consisted of middle-aged individuals (43-53 years).

The independent variable in all the articles was defined on the basis of the parents' occupation, except in the Chinese study, which used household assets during the individual's childhood. Waist circumference was analyzed as the outcome by nine articles $26,32,33,35,36,37,38,39,42$, five of which also evaluated WHR 32,33,35,36,42. In the four remaining articles, WHR was analyzed alone $34,40,41,43$. Only the Finnish article 35 and two British articles 36,42 evaluated hip circumference as outcome. All the articles presented their results separately for men and women, except the New Zealand study 41 .

Ninearticles reportedlosses rangingfrom $40 \%$ to $69 \%$ of the initial sample $32,33,34,35,36,37,40,41,43$, while in three others the losses were approximately $25 \% 38,39,42$. Only the article from China reported smaller losses $(10 \%$ in men and $1 \%$ in women) 26 . Six articles provided a detailed description of the losses $32,35,37,38,39,40$, and in two 
Selection process for studies evaluating the association between childhood socioeconomic status and abdominal obesity in adulthood (according to QUOROM guidelines).

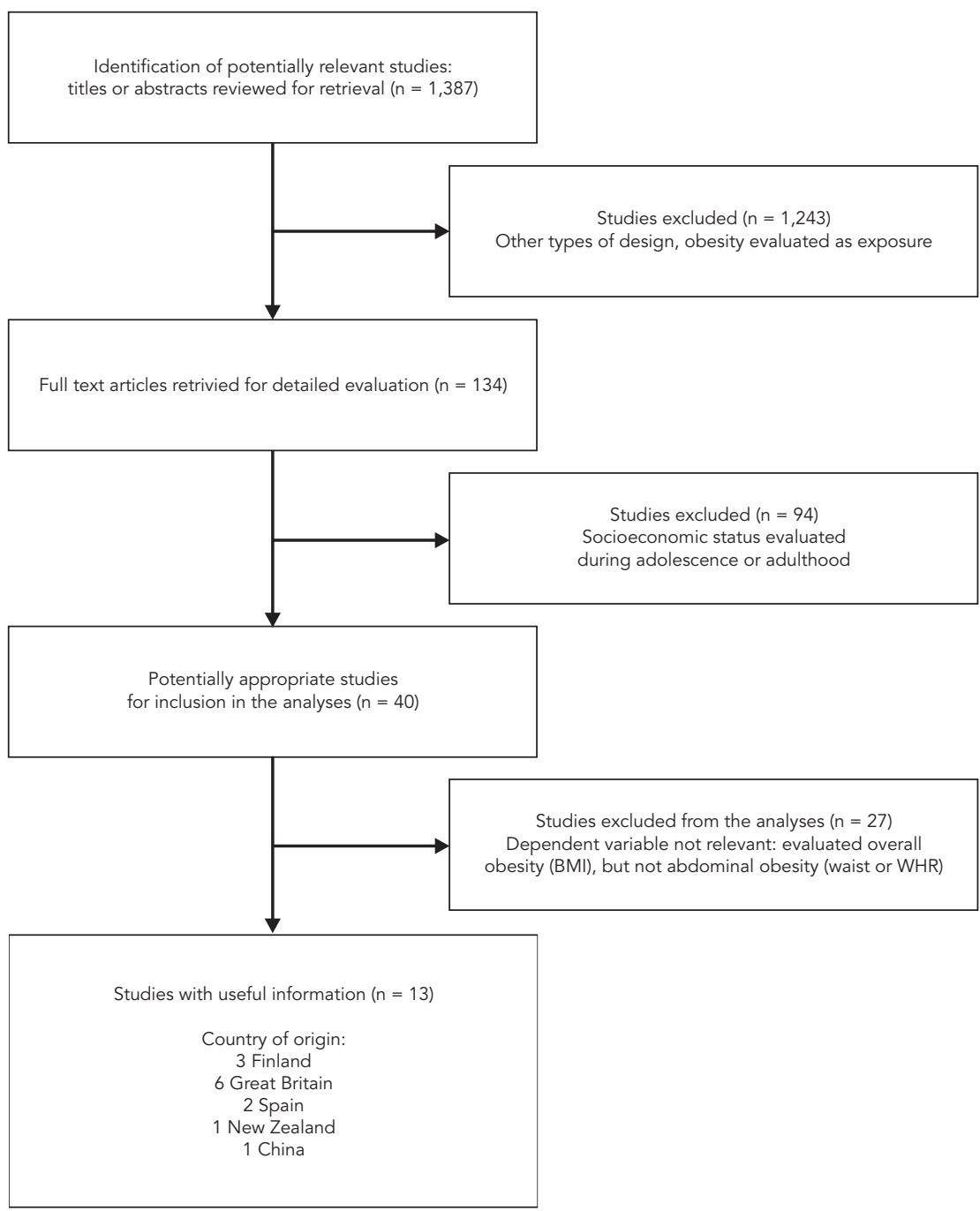

WHR: waist-hip ratio; BMI: body mass index.

articles they were more common among the poor 35,37 .

Table 1 summarizes the articles included in the review. The principal results will be discussed according to the target outcome.

\section{Waist circumference}

In women, the nine articles that evaluated the relationship between early socioeconomic status and waist circumference in adulthood found significant inverse associations 26,32,33,35,36,37,38,39,42.
This was observed both in articles where waist circumference was treated as a continuous variable $26,32,33,35,39,42$, as well as in those where it was treated as greater than or equal to $88 \mathrm{~cm} 37,38$. It was possible to calculate SII for six articles, all generating negative values. For five articles in which the outcome was continuous $26,32,35,36,39$, women from wealthy families in their childhood presented waist circumferences $2.2 \mathrm{~cm}$ (China) and $6.8 \mathrm{~cm}$ (Great Britain) smaller than in women from poor families. In the only study that used a categorical outcome, the index was 11.1 percent- 
Table 1

Studies that evaluated associations between childhood socioeconomic status and abdominal obesity in adulthood.

\begin{tabular}{|c|c|c|c|c|c|c|c|}
\hline \multirow[t]{2}{*}{ Reference } & \multirow[t]{2}{*}{ Country/Year } & \multirow[t]{2}{*}{ Design } & \multirow{2}{*}{$\begin{array}{c}\text { Sample } \\
\text { characteristics }\end{array}$} & \multirow[t]{2}{*}{ Exposure } & \multirow{2}{*}{$\begin{array}{l}\text { Outcome/Mean } \\
\text { (SD)/Adjustment }\end{array}$} & \multicolumn{2}{|c|}{ Results } \\
\hline & & & & & & Men & Women \\
\hline $\begin{array}{l}\text { Kivimaki } \\
\text { et al. } 32\end{array}$ & Finland/2006 & $\begin{array}{l}\text { Prospective } \\
\text { cohort of children } \\
\text { and adolescents } \\
\text { starting in } 1980 \\
\text { with retrospective } \\
\text { data on SES }\end{array}$ & $\begin{array}{c}\text { Born 1962-1977; } \\
\text { age: } 24-39 \\
\text { years; } 856 \text { men; } \\
\text { 1,066 women; } \\
\text { losses: } 55.5 \% ; \\
\text { larger proportion } \\
\text { of women in } \\
\text { relation to } \\
\text { original cohort }\end{array}$ & $\begin{array}{c}\text { Parents' } \\
\text { occupation } \\
\text { during childhood } \\
\text { or adolescence: } \\
\text { I: high non- } \\
\text { manual; II: low } \\
\text { non-manual; III: } \\
\text { manual. } \\
\text { Excluded } \\
\text { unemployed; } \\
\text { evaluated as } \\
\text { continuous } \\
\text { variable in } \\
\text { adjusted analysis }\end{array}$ & $\begin{array}{c}\text { Waist } \\
\text { M: } 89.8 \\
\text { W: } 79.7 \\
\text { WHR } \\
\text { M: } 0.90 \\
\text { W: } 0.79 \\
\text { Adjusted for age } \\
\text { and current SES }\end{array}$ & $\begin{array}{c}\text { Waist: Inverse } \\
\text { Crude: I: } 88.1 ; \text { II: } \\
\text { 89.5; III: } 90.2 \mathrm{~cm} \text {. } \\
\text { SII: }-2.6 \mathrm{~cm} \\
\text { (95\%Cl: }-4.4 ;-0.9) \\
\text { Adjusted: } \beta \text { for } \\
\text { trend } 1.0 \mathrm{~cm} . \mathrm{P} \\
=0.06 \\
\text { WHR: Inverse } \\
\text { Crude: I: } 0.88 ; \text { II: } \\
\text { 0.89; III: } 0.90 \mathrm{~cm} . \\
\text { SII: } 0.007 \text { (95\%Cl: } \\
\text {-1.083; } 1.885) \\
\text { Adjusted: } \beta \text { for } \\
\text { trend } 0.01 . \mathrm{p}= \\
0.006\end{array}$ & $\begin{array}{l}\text { Waist: Inverse } \\
\text { Crude: I: } 77.2 ; \text { II: } \\
\text { 79.2; III: } 80.7 \mathrm{~cm} \text {. } \\
\text { SII: }-4.5 \mathrm{~cm} \\
\text { (95\%Cl: }-6.3 ;-2.7 \text { ) } \\
\text { Adjusted: } \beta \text { for } \\
\text { trend } 1.2 \mathrm{~cm} . \mathrm{p} \\
=0.02 \\
\text { WHR: Inverse } \\
\text { Crude: I: } 0.78 \text {; II: } \\
\text { 0.79; III: } 0.80 \mathrm{~cm} \text {. } \\
\text { SII: }-0.027 \\
\text { (95\%Cl: }-0.032 ; \\
-0.021) \\
\text { Adjusted: } \beta \text { for } \\
\text { trend } 0.01 . \mathrm{p}< \\
0.001\end{array}$ \\
\hline $\begin{array}{l}\text { Laitinen } \\
\text { et al. } 34\end{array}$ & Finland/2004 & $\begin{array}{c}\text { Prospective birth } \\
\text { cohort }\end{array}$ & $\begin{array}{c}\text { Born 1966; } \\
\text { age: } 31 \text { years; } \\
2,841 \text { men; } \\
2,930 \text { women; } \\
\text { losses: } 52.1 \% ; \\
\text { differences not } \\
\text { specified }\end{array}$ & $\begin{array}{l}\text { Father's } \\
\text { occupation } \\
\text { at birth: I: } \\
\text { professionals; II: } \\
\text { skilled workers; } \\
\text { III: unskilled } \\
\text { workers; IV: } \\
\text { farmers }\end{array}$ & $\begin{array}{l}\text { WHR } \\
\text { Evaluated as } \\
\text { prevalence } \\
\text { of abdominal } \\
\text { obesity (> 90th } \\
\text { percentile) } \\
\text { Not adjusted }\end{array}$ & $\begin{array}{c}\text { WHR: No } \\
\text { association } \\
\text { Crude: I: } 8 \% \text {; II: } \\
\text { 11\%; III: } 9 \% \text {; IV: } \\
\text { 11\%. p }=0.09 \text {. } \\
\text { SII: }-2.5 \text { p.p. } \\
\text { (95\%Cl: }-7.9 ; 3.0)\end{array}$ & $\begin{array}{l}\text { WHR: Inverse } \\
\text { association } \\
\text { Crude: I: } 9 \% \text {; II: } \\
9 \% \text {; III: } 13 \% \text {; IV: } \\
\text { 10\%. p }=0.01 \\
\text { SII: }-3.2 \text { p.p. } \\
\text { (95\%Cl: }-10.1 ;- \\
\text { 3.7) }\end{array}$ \\
\hline $\begin{array}{l}\text { Laitinen } \\
\text { et al. } 35\end{array}$ & Finland/2001 & $\begin{array}{c}\text { Prospective birth } \\
\text { cohort }\end{array}$ & $\begin{array}{c}\text { Born 1966; age: } \\
31 \text { years; } 2,876 \\
\text { men; 3,404 } \\
\text { women; losses: } \\
47.9 \% ; \text { more } \\
\text { losses among } \\
\text { poor and high } \\
\text { BMI }\end{array}$ & $\begin{array}{c}\text { Father's } \\
\text { occupation } \\
\text { at birth: I/II: } \\
\text { higher-paid and } \\
\text { better-educated } \\
\text { professionals; III: } \\
\text { skilled workers; } \\
\text { IV: unskilled } \\
\text { workers; V: } \\
\text { farmers }\end{array}$ & $\begin{array}{c}\text { Waist } \\
\text { M: } 89.0(10.0) \\
\text { W: } 79.0(12.0) \\
\text { Hip } \\
\text { Mean not } \\
\text { included } \\
\text { WHR } \\
\text { M: } 0.91(0.06) \\
\text { W: } 0.81(0.08) \\
\text { Not adjusted }\end{array}$ & $\begin{array}{c}\text { Waist: No } \\
\text { association } \\
\text { I/II: 88.0; III: } \\
\text { 89.0; IV: 88.0; } \\
\text { V: 89.0cm. SII: } \\
-0.7 \mathrm{~cm}(95 \% \mathrm{Cl} \text { : } \\
\text {-3.0;1.7) } \\
\text { Hip: No } \\
\text { association } \\
\text { Mean 97.0cm in } \\
\text { all categories. SII: } \\
\text { 0.0cm (95\%Cl: } \\
\text { 0.0;0.0) } \\
\text { WHR: Inverse } \\
\text { Categories I/II/III/ } \\
\text { IV: } 0.91 ; \mathrm{V}: 0.92 . \\
\text { p = } 0.04 . \mathrm{SII}: \\
-0.010(95 \% \mathrm{Cl} \text { : } \\
\text {-0.024; } 0.004)\end{array}$ & $\begin{array}{c}\text { Waist: Inverse } \\
\text { I/II/III: 78.0; IV: } \\
\text { 80.0; V: 79.0cm. } \\
\text { p < 0.001. SII: } \\
\text {-2.6cm (95\%Cl: } \\
\text {-4.2;-1.0) } \\
\text { Hip: No } \\
\text { association } \\
\text { I/II/III: } 97.0 ; \text { IV: } \\
\text { 98.0; V: 97.0cm. } \\
\text { SII: -0.6cm } \\
\text { (95\%Cl: }-2.6 ; 1.4) \\
\text { WHR: Inverse } \\
\text { I/II: 0.80; III: 0.81; } \\
\text { IV: } 0.82 ; \mathrm{V}: 0.81 . \\
\text { P < } 0.001 . \mathrm{SII} \\
-0.018(95 \% \mathrm{Cl}: \\
-0.042 ; 0.007)\end{array}$ \\
\hline \multirow[t]{2}{*}{$\begin{array}{l}\text { Langen- } \\
\text { berg et } \\
\text { al. } 37\end{array}$} & \multirow[t]{2}{*}{$\begin{array}{c}\text { Great } \\
\text { Britain/2006 }\end{array}$} & \multirow[t]{2}{*}{$\begin{array}{l}\text { Prospective birth } \\
\text { cohort }\end{array}$} & \multirow{2}{*}{$\begin{array}{c}\text { Born 1946; age: } \\
53 \text { years; } 1,311 \\
\text { men; } 1,318 \\
\text { women; losses: } \\
51.0 \% ; \text { more } \\
\text { losses among } \\
\text { poor }\end{array}$} & \multirow{2}{*}{$\begin{array}{c}\text { Father's } \\
\text { occupation when } \\
\text { individual was } \\
4 \text { years of age: } \\
\text { I: professional; } \\
\text { II: middle-level; } \\
\text { IIla: skilled non- } \\
\text { manual; Illb: } \\
\text { skilled manual; } \\
\text { IV: semi- skilled; } \\
\text { V: unskilled }\end{array}$} & \multirow{2}{*}{$\begin{array}{c}\text { Waist } \\
\text { Evaluated as } \\
\text { prevalence } \\
\text { of abdominal } \\
\text { obesity } \\
M>102 \mathrm{~cm} \\
W>88 \mathrm{~cm} \\
\text { Adjusted for } \\
\text { own schooling } \\
\text { and current } \\
\text { occupation }\end{array}$} & \multicolumn{2}{|c|}{$\begin{array}{l}\text { Socioeconomic status transformed } \\
\text { into values between } 0 \text { (wealthier - } \\
\text { reference group) and } 1 \text { (poorer) to } \\
\text { present results as RII (relative index of } \\
\text { inequality); interpretable as relative risk }\end{array}$} \\
\hline & & & & & & $\begin{array}{c}\text { Waist: No } \\
\text { association } \\
\text { Crude: RII: } 1.4 \\
\text { (95\%Cl: } 0.9-2.1 \text { ) } \\
\text { Adjusted: RII: } 1.1 \\
\text { (95\%Cl: } 0.7-1.7 \text { ) }\end{array}$ & $\begin{array}{l}\text { Waist: Inverse } \\
\text { Crude: RII: } 2.4 \\
\text { (95\%Cl: } 1.6-3.5) \text {. } \\
\text { Adjusted: RII: } 1.8 \\
\text { (95\%Cl: } 1.1-2.8)\end{array}$ \\
\hline
\end{tabular}

(continues) 
Table 1 (continued)

\begin{tabular}{|c|c|c|c|c|c|c|c|}
\hline \multirow[t]{2}{*}{ Reference } & \multirow[t]{2}{*}{ Country/Year } & \multirow[t]{2}{*}{ Design } & \multirow{2}{*}{$\begin{array}{l}\text { Sample } \\
\text { characteristics }\end{array}$} & \multirow[t]{2}{*}{ Exposure } & \multirow{2}{*}{$\begin{array}{l}\text { Outcome/Mean } \\
\text { (SD)/Adjustment }\end{array}$} & \multicolumn{2}{|c|}{ Results } \\
\hline & & & & & & Men & Women \\
\hline \multirow[t]{3}{*}{$\begin{array}{l}\text { Langen- } \\
\text { berg et } \\
\text { al. } 36\end{array}$} & $\begin{array}{l}\text { Great } \\
\text { Britain/2003 }\end{array}$ & $\begin{array}{l}\text { Prospective birth } \\
\text { cohort }\end{array}$ & $\begin{array}{l}\text { Born 1946; } \\
\text { age: } 53 \text { years; } \\
\text { 1,472 men; } \\
\text { 1,563 women; } \\
\text { losses: } 43.4 \% ; \text { no } \\
\text { differences from } \\
\text { original cohort } \\
\text { (comparison } \\
\text { variables not } \\
\text { specified) }\end{array}$ & $\begin{array}{c}\text { Father's } \\
\text { occupation when } \\
\text { individual was } \\
4 \text { years of age: } \\
\text { I: professional; } \\
\text { II: middle-level; } \\
\text { IIla: skilled non- } \\
\text { manual; Illb: } \\
\text { skilled manual; } \\
\text { IV: semi-skilled; } \\
\text { V: unskilled. } \\
\text { Change in SES: } \\
\text { from childhood } \\
\text { to } 43 \text { years } \\
\text { (by head-of- } \\
\text { household's } \\
\text { occupation, } \\
\text { categorized } \\
\text { like father's } \\
\text { occupation) }\end{array}$ & $\begin{array}{c}\text { Waist } \\
\text { M: } 97.7(10.8) \\
\text { W: } 85.7(12.9) \\
\text { Hip } \\
\text { M: } 14.2(7.1) \\
\text { W: } 106.1(11.0) \\
\text { WHR } \\
\text { M: } 0.94(0.06) \\
\text { W: } 0.81 \text { (0.07) } \\
\text { Adjusted for SES } \\
\text { in other periods. } \\
\text { Change in SES } \\
\text { adjusted for } \\
\text { physical activity } \\
\text { and current } \\
\text { smoking }\end{array}$ & 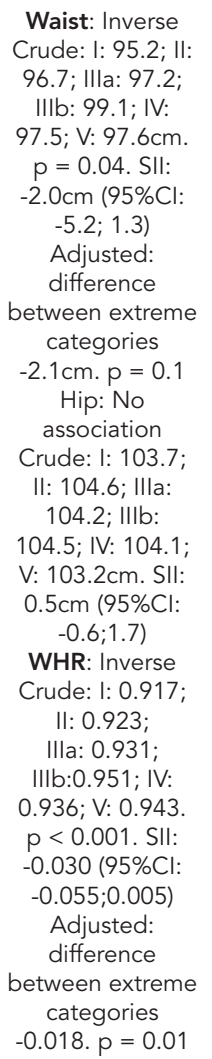 & 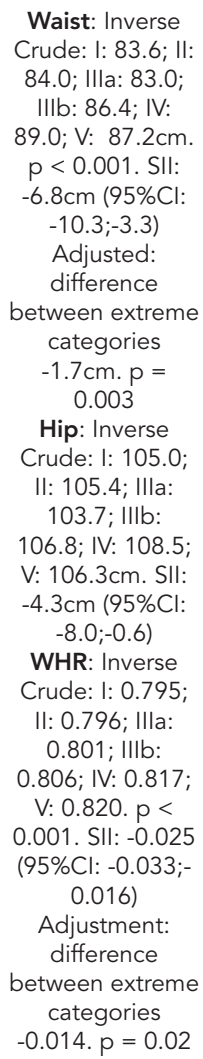 \\
\hline & & & & & & \multicolumn{2}{|c|}{ Change in SES } \\
\hline & & & & & & $\begin{array}{c}\text { Smaller waist } \\
\text { and WHR among } \\
\text { those who were } \\
\text { always classes } \\
\text { I-II and larger } \\
\text { among always } \\
\text { IIlb }\end{array}$ & $\begin{array}{c}\text { Smaller waist } \\
\text { and WHR among } \\
\text { those who were } \\
\text { always classes } \\
\text { I-II and larger } \\
\text { among always } \\
\text { IV-V }\end{array}$ \\
\hline $\begin{array}{l}\text { Kuh et } \\
\text { al. } 33\end{array}$ & $\begin{array}{l}\text { Great } \\
\text { Britain/2002 }\end{array}$ & $\begin{array}{l}\text { Prospective birth } \\
\text { cohort }\end{array}$ & $\begin{array}{l}\text { Born 1946; } \\
\text { age: } 43 \text { years; } \\
\text { 1,634 men; } \\
\text { 1,632 women; } \\
\text { losses: } 39 \% ; \text { no } \\
\text { differences from } \\
\text { original cohort } \\
\text { (comparison } \\
\text { variables not } \\
\text { specified) }\end{array}$ & $\begin{array}{c}\text { Father's } \\
\text { occupation } \\
\text { in childhood: } \\
\text { manual; } \\
\text { non- manual } \\
\text { (reference group) } \\
\text { When father's } \\
\text { occupation } \\
\text { unknown, } \\
\text { household } \\
\text { crowding ( } \geq 2 \\
\text { persons per } \\
\text { room) was used } \\
\text { to indicate } \\
\text { poverty }\end{array}$ & $\begin{array}{c}\text { Waist } \\
\text { M: } 91.9(9.8) \\
\text { W: } 77.7(11.1) \\
\text { Hip } \\
\text { M: } 100.6(7.1) \\
\text { W: } 100.5(10.1) \\
\text { WHR } \\
\text { M: } 0.91(0.06) \\
\text { W: } 0.77(0.06) \\
\text { Adjusted for } \\
\text { birth, weight at } 7 \\
\text { years, BMI, and } \\
\text { SES in adulthood }\end{array}$ & $\begin{array}{c}\text { Waist: Inverse } \\
\text { Crude: } \beta \text { : } \\
\text { 2.14cm. } p< \\
0.001 \\
\text { Adjusted: } \beta \text { : } \\
\text { 0.84. } p=0.02 \\
\text { Hip: not } \\
\text { evaluated } \\
\text { WHR: Inverse } \\
\text { Crude: } \beta \text { : } 0.018 \text {. } \\
\text { p < } 0.001 \\
\text { Adjusted: } \beta \text { : } \\
\text { 0.056. } p=0.05\end{array}$ & $\begin{array}{c}\text { Waist: Inverse } \\
\text { Crude: } \beta \text { : } \\
3.57 \mathrm{~cm} . \mathrm{p}< \\
0.001 \\
\text { Adjusted: } \beta \text { : } \\
\text { 0.35. } \mathrm{p}=0.3 \\
\text { Hip: not } \\
\text { evaluated } \\
\text { WHR: Inverse } \\
\text { Crude: } \beta \text { : } 0.033 \text {. } \\
\text { p < } 0.001 \\
\text { Adjusted: } \beta \text { : } \\
\text { 0.006. } p=0.05\end{array}$ \\
\hline \multirow[t]{2}{*}{$\begin{array}{l}\text { Parker et } \\
\text { al. } 40\end{array}$} & $\begin{array}{l}\text { Great } \\
\text { Britain/2003 }\end{array}$ & $\begin{array}{l}\text { Prospective birth } \\
\text { cohort }\end{array}$ & $\begin{array}{l}\text { Born 1947; } \\
\text { age: } 49-51\end{array}$ & $\begin{array}{l}\text { Father's } \\
\text { occupation }\end{array}$ & $\begin{array}{c}\text { WHR } \\
\text { M: } 0.95(0.06)\end{array}$ & \multicolumn{2}{|c|}{$\begin{array}{l}\text { Show coefficients for trend and not by } \\
\text { exposure category }\end{array}$} \\
\hline & & & $\begin{array}{l}\text { years; } 154 \text { men; } \\
204 \text { women; } \\
\text { losses: } 68.7 \% ; \\
\text { no differences } \\
\text { according to } \\
\text { birth weight or } \\
\text { SES }\end{array}$ & $\begin{array}{l}\text { at birth: I: } \\
\text { professional; II: } \\
\text { administrative; } \\
\text { III: skilled; IV: } \\
\text { semi-skilled; V: } \\
\text { unskilled }\end{array}$ & $\begin{array}{l}\text { W: } 0.80(0.06) \\
\text { Not adjusted }\end{array}$ & $\begin{array}{c}\text { WHR: Direct } \\
\text { Crude: } \beta \text { : }-0.016 . \\
\quad p=0.001\end{array}$ & $\begin{array}{c}\text { WHR: No } \\
\text { association } \\
\text { Crude: } \beta \text { : }-0.005 \text {. } \\
\quad p=0.2\end{array}$ \\
\hline
\end{tabular}

(continues) 
Table 1 (continued)

\begin{tabular}{|c|c|c|c|c|c|c|c|}
\hline \multirow[t]{2}{*}{ Reference } & \multirow[t]{2}{*}{ Country/Year } & \multirow[t]{2}{*}{ Design } & \multirow{2}{*}{$\begin{array}{c}\text { Sample } \\
\text { characteristics }\end{array}$} & \multirow[t]{2}{*}{ Exposure } & \multirow{2}{*}{$\begin{array}{l}\text { Outcome/Mean } \\
\text { (SD)/Adjustment }\end{array}$} & \multicolumn{2}{|c|}{ Results } \\
\hline & & & & & & Men & Women \\
\hline $\begin{array}{l}\text { Lawlor et } \\
\text { al. } 43\end{array}$ & $\begin{array}{c}\text { Great } \\
\text { Britain/2002 }\end{array}$ & $\begin{array}{l}\text { Population } \\
\text { survey with } \\
\text { retrospective } \\
\text { information on } \\
\text { SES (women) }\end{array}$ & $\begin{array}{c}\text { Born 1920-1940; } \\
\text { age: } 60-79 \text { years; } \\
\text { 4,286 women; } \\
\text { losses: } 40 \% ; \\
\text { more losses } \\
\text { among smokers, } \\
\text { higher WHR, and } \\
\text { more elderly }\end{array}$ & $\begin{array}{c}\text { Father's } \\
\text { occupation } \\
\text { at birth: I: } \\
\text { professional; II: } \\
\text { middle-level; } \\
\text { IIla: skilled non- } \\
\text { manual; IIlb: } \\
\text { skilled manual; } \\
\text { IV: semi-skilled; } \\
\text { V: unskilled. } \\
\text { Change in SES: } \\
\text { from manual } \\
\text { (IIIb, IV, and V) to } \\
\text { non-manual (I, II, } \\
\text { and IIla) between } \\
\text { childhood and } \\
\text { adulthood (own } \\
\text { occupation or } \\
\text { that of husband) }\end{array}$ & $\begin{array}{c}\text { WHR } \\
\text { W: } 0.818 \\
\text { Adjustment 1: } \\
\text { age } \\
\text { Adjustment 2: } \\
\text { age and current } \\
\text { occupation } \\
\text { For change in } \\
\text { SES, outcome } \\
\text { was prevalence } \\
\text { of abdominal } \\
\text { obesity (WHR } \geq \\
\text { 0.85). }\end{array}$ & & 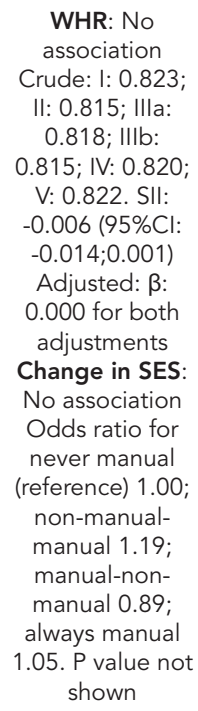 \\
\hline \multirow[t]{2}{*}{$\begin{array}{l}\text { Brunner } \\
\text { et al. } 42\end{array}$} & \multirow[t]{2}{*}{$\begin{array}{c}\text { Great } \\
\text { Britain/1999 }\end{array}$} & \multirow{2}{*}{$\begin{array}{l}\text { Population } \\
\text { survey with } \\
\text { retrospective } \\
\text { information on } \\
\text { SES }\end{array}$} & \multirow{2}{*}{$\begin{array}{c}\text { Born 1930-1953; } \\
\text { age: } 60-79 \text { years; } \\
\text { 4,774 men; } 2,206 \\
\text { women; losses: } \\
27 \% ; \text { no report } \\
\text { on differences } \\
\text { between sample } \\
\text { and losses }\end{array}$} & \multirow{2}{*}{$\begin{array}{l}\text { Father's } \\
\text { occupation } \\
\text { at birth: I/II: } \\
\text { professional and } \\
\text { middlle-level; } \\
\text { Illa: skilled non- } \\
\text { manual; Illb: } \\
\text { skilled manual; } \\
\text { IV/V: semi-skilled } \\
\text { and unskilled }\end{array}$} & \multirow{2}{*}{$\begin{array}{c}\text { Waist, Hip, } \\
\text { WHR: Means not } \\
\text { shown } \\
\text { Adjustment for } \\
\text { age }\end{array}$} & \multicolumn{2}{|c|}{$\begin{array}{c}\text { Shows differences between extreme } \\
\text { categories of SES, without crude } \\
\text { results }\end{array}$} \\
\hline & & & & & & 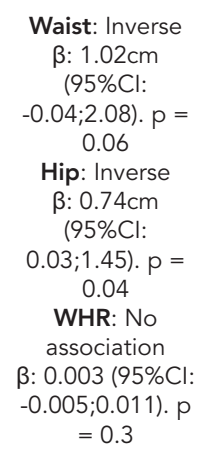 & 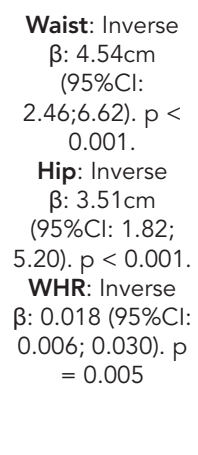 \\
\hline $\begin{array}{l}\text { Regidor } \\
\text { et al. } 39\end{array}$ & Spain/2004 & $\begin{array}{l}\text { Population } \\
\text { survey with } \\
\text { retrospective } \\
\text { information on } \\
\text { SES }\end{array}$ & $\begin{array}{c}\text { Born before } \\
\text { 1940; age: mean } \\
71.9 \text { years; } \\
\text { 1,660 men; } \\
\text { 1,998 women; } \\
\text { losses: } 24 \% ; \\
\text { no differences } \\
\text { by age, sex, or } \\
\text { schooling }\end{array}$ & $\begin{array}{c}\text { Father's } \\
\text { occupation } \\
\text { in childhood: } \\
\text { I: higher } \\
\text { professionals, } \\
\text { directors, clergy; } \\
\text { II: farm owners; } \\
\text { III: skilled } \\
\text { and unskilled } \\
\text { manual; IV: farm } \\
\text { employees }\end{array}$ & $\begin{array}{c}\text { Waist } \\
\text { M: } 106.0 \\
\text { W: } 110.5 \\
\text { Adjustment 1: } \\
\text { age } \\
\text { Adjustment 2: } \\
\text { age, current } \\
\text { occupation, } \\
\text { and individual's } \\
\text { schooling }\end{array}$ & $\begin{array}{c}\text { Waist: "U"- } \\
\text { shaped } \\
\text { association } \\
\text { Crude: I: } 106.1 \text {; } \\
\text { II: } 105.4 ; \text { III: } \\
\text { 105.6; IV: } \\
\text { 107.1 cm. SII: } \\
-0.9 \mathrm{~cm}(95 \% \mathrm{Cl} \text { : } \\
-3.7 ; 1.9) \\
\text { Adjusted 1: } \beta \\
\text { class I: } 0.0 ; \text { II: } \\
-0.78 ; \text { III:-0.67; IV: } \\
0.95 . \\
\text { Adjusted 2: } \\
\text { reduction in } \\
\text { coefficients } \\
\text { and loss of } \\
\text { significance }\end{array}$ & $\begin{array}{l}\text { Waist: Inverse } \\
\text { Crude: I: 110.0; } \\
\text { II: } 109.9 ; \text { III: } \\
110.4 ; \text { IV: } \\
112.3 \mathrm{~cm} . \mathrm{SII}: \\
-2.5 \mathrm{~cm}(95 \% \mathrm{Cl} \text { : } \\
-5.1 ; 0.2) \\
\text { Adjusted } 1: \beta \\
\text { class I: } 0.0 ; \text { II: } \\
-0.02 ; \text { III: } 0.16 ; \text { IV: } \\
2.11) \\
\text { Adjusted } 2 \text { : } \\
\text { reduction in } \\
\text { coefficients } \\
\text { and loss of } \\
\text { significance }\end{array}$ \\
\hline
\end{tabular}

(continues) 
Table 1 (continued)

\begin{tabular}{|c|c|c|c|c|c|c|c|}
\hline \multirow[t]{2}{*}{ Reference } & \multirow[t]{2}{*}{ Country/Year } & \multirow[t]{2}{*}{ Design } & \multirow{2}{*}{$\begin{array}{c}\text { Sample } \\
\text { characteristics }\end{array}$} & \multirow[t]{2}{*}{ Exposure } & \multirow{2}{*}{$\begin{array}{l}\text { Outcome/Mean } \\
\text { (SD)/Adjustment }\end{array}$} & \multicolumn{2}{|c|}{ Results } \\
\hline & & & & & & Men & Women \\
\hline \multirow[t]{2}{*}{$\begin{array}{l}\text { Regidor } \\
\text { et al. } 38\end{array}$} & \multirow[t]{2}{*}{ Spain/2004 } & \multirow[t]{2}{*}{$\begin{array}{l}\text { Population } \\
\text { survey with } \\
\text { retrospective } \\
\text { information on } \\
\text { SES }\end{array}$} & \multirow[t]{2}{*}{$\begin{array}{c}\text { Born before } \\
\text { 1940; age: mean } \\
\text { 71.9 years; } \\
\text { 1,660 men; } \\
\text { 1,998 women; } \\
\text { losses: } 24 \% ; \\
\text { no differences } \\
\text { by age, sex, or } \\
\text { schooling }\end{array}$} & \multirow{2}{*}{$\begin{array}{c}\text { Father's } \\
\text { occupation } \\
\text { in childhood: } \\
\text { I: higher } \\
\text { professionals, } \\
\text { directors, clergy; } \\
\text { II: farm owners; } \\
\text { III: skilled } \\
\text { and unskilled } \\
\text { manual; IV: farm } \\
\text { employees. } \\
\text { Change in } \\
\text { SES: between } \\
\text { childhood and } \\
\text { adulthood. } \\
\text { Father's } \\
\text { occupation } \\
\text { and current } \\
\text { occupation } \\
\text { divided into non- } \\
\text { manual (I and II) } \\
\text { and manual (III } \\
\text { and IV) }\end{array}$} & \multirow[t]{2}{*}{$\begin{array}{c}\text { Waist } \\
\text { Evaluated as } \\
\text { prevalence } \\
\text { of abdominal } \\
\text { obesity } \\
M>102 \mathrm{~cm} \\
W>88 \mathrm{~cm} \\
\text { Adjusted for } \\
\text { age and current } \\
\text { occupation }\end{array}$} & $\begin{array}{l}\text { Waist: "U"- } \\
\text { shaped } \\
\text { association } \\
\text { Crude: I: } 49.5 \% \text {; } \\
\text { II: } 48.7 \% \text {; III: } \\
\text { 43.7\%; IV: } \\
55.6 \% \text {. p for } \\
\text { heterogeneity } \\
\text { =0.02. SII: }-3.0 \\
\text { p.p. (95\%Cl: } \\
\text {-22.1;16.1) } \\
\text { Adjusted: } \\
\text { compared to } \\
\text { class I, risk was } \\
\text { 14\% lower in } \\
\text { class III and } 11 \% \\
\text { higher in class IV } \\
\text { Chang }\end{array}$ & $\begin{array}{l}\text { Waist: Inverse } \\
\text { Crude: I: } 76.0 \% \text {; } \\
\text { II: } 76.7 \% \text {; III: } \\
\text { 78.5\%; IV: } \\
86.1 \% . \mathrm{P}= \\
\text { 0.01. SII: }-11.1 \\
\text { p.p. }(95 \% \mathrm{Cl} \text { : } \\
\text {-20.6;1.6) } \\
\text { Adjusted: } \\
\text { compared to } \\
\text { class I, risk was } \\
\text { 20\% greater in } \\
\text { class IV }\end{array}$ \\
\hline & & & & & & No association & $\begin{array}{l}\text { Prevalence } \\
10 \% \text { higher in } \\
\text { non-manual- } \\
\text { manual and } \\
\text { always manual } \\
\text { categories } \\
\text { compared to } \\
\text { other groups }\end{array}$ \\
\hline $\begin{array}{l}\text { Poulton } \\
\text { et al. } 41\end{array}$ & $\begin{array}{c}\text { New } \\
\text { Zealand/2002 }\end{array}$ & $\begin{array}{c}\text { Prospective birth } \\
\text { cohort }\end{array}$ & $\begin{array}{l}\text { Born 1972- } \\
\text { 1973; age: } 26 \\
\text { years; } 154 \text { men; } \\
204 \text { women; } \\
\text { losses: } 65.5 \% ; \\
\text { differences not } \\
\text { specified }\end{array}$ & $\begin{array}{l}\text { Parents' } \\
\text { occupation } \\
\text { during childhood } \\
\text { and adolescence: } \\
\text { high, medium, } \\
\text { and low SES } \\
\text { (mean SES } \\
\text { at different } \\
\text { visits during } \\
\text { childhood and } \\
\text { adolescence). } \\
\text { Excludes } \\
\text { students and } \\
\text { unemployed. } \\
\text { Change in } \\
\text { SES: between } \\
\text { childhood and } \\
\text { adulthood }\end{array}$ & $\begin{array}{c}\text { WHR } \\
\text { Descriptive } \\
\text { analysis not } \\
\text { shown } \\
\text { Adjusted for sex, } \\
\text { childhood health } \\
\text { conditions, and } \\
\text { SES in adulthood }\end{array}$ & $\begin{array}{r}\text { Results not s } \\
\text { WHR } \\
\text { Crude: low SES } 0 \\
\text { high } \\
\text { Adjusted: } \beta \text { for lo } \\
0.004 ; \text { high } \\
\text { Chanc } \\
\text { Higher WHR a } \\
\text { whose SES was } \\
\text { intermediate valu } \\
\text { improved their SE } \\
\text { among those with } \\
\text { consistently high }\end{array}$ & $\begin{array}{l}\text { atified by sex } \\
\text { verse } \\
1 ; \text { medium } 0.799 ; \\
793 \text {. } \\
\text { SES } 0.0 ; \text { medium } \\
\text { 16. } \mathrm{p}=0.002 \\
\text { in SES } \\
\text { ong individuals } \\
\text { ways low }(0.82) ; \\
\text { among those who } \\
(0.81) ; \text { lower WHR } \\
\text { vorsened SES and } \\
\text { S }(0.80) . p=0.03\end{array}$ \\
\hline $\begin{array}{l}\text { Schooling } \\
\text { et al. } 26\end{array}$ & China/2008 & $\begin{array}{l}\text { Cohort of adults } \\
\text { with retrospective } \\
\text { information on } \\
\text { SES }\end{array}$ & $\begin{array}{c}\text { Born 1911-1955; } \\
\text { age: } 50-94 \text { years; } \\
\text { 2,735 men; } \\
7,011 \text { women; } \\
\text { losses: } 10 \% \text { men } \\
\text { and } 1 \% \text { women; } \\
\text { differences not } \\
\text { specified }\end{array}$ & $\begin{array}{l}\text { Household assets } \\
\text { in childhood: } \\
\text { clock, bicycle, } \\
\text { and sewing } \\
\text { machine. } \\
\text { Number of items } \\
\text { categorized as } \\
\text { as: } 0 ; 1-2 ; 3 \text { items }\end{array}$ & $\begin{array}{c}\text { Waist } \\
\text { M: } 81.0 \\
\text { W: } 76.1 \\
\text { Adjustment 1: } \\
\text { age, smoking, } \\
\text { alcohol, physical } \\
\text { activity, and } \\
\text { height } \\
\text { Adjustment 2: } \\
\text { as previously + } \\
\text { current SES }\end{array}$ & $\begin{array}{l}\text { Waist: Direct } \\
\text { Crude: } 0 \text { items: } \\
\text { 80.7; } 1-2: 80.8 ; \\
\text { 3: } 82.2 \mathrm{~cm} . p< \\
0.01 . \mathrm{SII}: 2.8 \mathrm{~cm} \\
\text { (95\% } \mathrm{Cl}: 1.9 ; 3.7) \\
\text { Adjustment } 1: \beta \text { : } \\
\text { 1.4cm }(p=0.01) \\
\text { Adjustment } 2: \\
1.3 \mathrm{~cm}(p=0.02)\end{array}$ & $\begin{array}{l}\text { Waist: Inverse } \\
\text { Crude: } 0 \text { items: } \\
\text { 76.8; } 1-2: 75.3 ; \\
\text { 3:75.2cm. } p< \\
0.01 . \mathrm{SIl}:-2.2 \mathrm{~cm} \\
\text { (95\%Cl: }-4.9 ; 0.5) \\
\text { Adjustment } 1: \beta \text { : } \\
-1.0 \mathrm{~cm}(p<0.01) \\
\text { Adjustment } 2 \text { : } \\
-0.3 \mathrm{~cm}(p=0.1)\end{array}$ \\
\hline
\end{tabular}

Note: waist and hip circumference in centimeters $(\mathrm{cm})$.

SES: socioeconomic status; $\beta$ : regression coefficient; M: men; W: women; WHR: waist-hip ratio; BMI: body mass index; SII: slope index of inequality. 
age points, once again with a lower prevalence among the wealthier 38 .

Current socioeconomic status was included as a co-variable in six articles: three showed a reduction in the coefficients with persistence of the association $32,36,37$, in two the relationship disappeared completely, 26,39 and one showed a slight increase in the effect measure 38 . In Kuh et al. 33, in the 1946 British cohort, current socioeconomic status was included with weight at seven years and current BMI, which led to complete loss of the original association.

In the nine articles with data on men $26,32,33,35,36,37,38,39,42$, the pattern in the associations was not as clear, nor was the magnitude of effects as pronounced as in women. Three articles on the 1946 British cohort 33,36,37, one British survey 42 , and a Finnish article 32 showed inverse associations between childhood socioeconomic status and waist circumference. Two of these articles calculated the SII 32,36, presenting values of $-2.0 \mathrm{~cm}$ (Great Britain) and $-2.6 \mathrm{~cm}$ (Finland). The two Spanish articles showed a non-linear relationship, and waist circumference was larger among elderly individuals belonging to the extreme categories of childhood socioeconomic status, especially the poorer 38,39 . Meanwhile there was a direct relationship in Chinese elderly (SII equal to $2.8 \mathrm{~cm}$ ) 26 . Only one article, with data from Finland 35 , found no association.

When current socioeconomic status was included in the analyses, two articles showed no change in the associations 26,39 , in two there was a reduction in the coefficients 32,36 , and in two others the association disappeared 37,38. In Kuh et al. 33 , with the inclusion of anthropometric variables (in addition to current socioeconomic status) there was a reduction in the coefficients, but the association remained significant.

\section{Hip circumference}

Only three articles evaluated the association between childhood socioeconomic status and hip circumference in adulthood. In Finland, there was no difference between the various categories of socioeconomic status for either men or women 35 . Meanwhile, two British articles found an inverse relationship, particularly in women 36,42 . In two of these articles it was possible to calculate the SII 35,36 , ranging from -0.6 to $-4.30 \mathrm{~cm}$ in women and 0.0 to $0.5 \mathrm{~cm}$ in men. None of the articles included adjusted analyses.

\section{WHR}

In women, the three Finnish articles 32,34,35 and three other British articles 33,36,42 found inverse associations between childhood socioeconomic status and WHR. In four articles it was possible to calculate the SII $32,34,35,36$, all of which showed negative values. In three, WHR was analyzed as a continuous variable $32,35,36$, with the SII ranging from -0.018 to -0.027 . In the article that treated WHR as a categorical variable, the SII was -3.2 p.p. ${ }^{34}$. Two other British studies 40,43 showed no relationship between childhood socioeconomic status and WHR in adulthood.

In men, of the seven articles that evaluated WHR as the outcome, four reported significant inverse associations $32,33,35,36$, two found no relationship 34,42 , and one found a direct association ${ }^{40}$. In three articles it was possible to calculate SII, two of which showed negative values $(-0.010$ and -0.025$) 35,36$. In the study by Kivimaki et al. 32 , the authors reported a significant inverse association after adjusting for age and current socioeconomic status, but the SII calculated from the crude data was not significant $(\mathrm{SII}=0.007$ ).

In the study from New Zealand, in which the analyses were performed without stratifying by gender, there was an inverse relationship between early socioeconomic status and WHR in adulthood 41 .

In three articles the analyses were adjusted for current socioeconomic status $32,36,41$, and in both men and women the associations remained significant despite the drop in the coefficients. The same occurred in Kuh et al. 33 when adult anthropometric variables and current socioeconomic status were included in the analyses.

\section{Other analyses}

Three studies assessed the association between change in socioeconomic status between childhood and adulthood and abdominal obesity. A study from the United Kingdom 36 and another from New Zealand ${ }^{41}$ showed a higher risk of abdominal obesity in men and women that had always been poor as compared to the non-poor. Meanwhile, a British survey of elderly women showed no association 43 .

\section{Discussion}

Except for a study in China 26, the articles reviewed here are from high-income countries. Most of the analyses show that among women, childhood poverty is associated with increases in waist circumference, hip circumference, and/ 
or WHR in adulthood. For men, the associations were weaker and did not show such a clear pattern as in women. Most of the authors found increases in waist circumference and WHR in men that were poor in childhood, but some studies found no association, and in still others the mean values were higher among the wealthy. This suggests that the influence of socioeconomic conditions on obesity is more evident and occurs earlier in women than in men.

The current findings are similar to those reported in the review by Parsons et al. 23 , with a predominance of inverse associations between childhood socioeconomic status and BMI in adults of both sexes, although no direct association was reported. Part of the difference in the results in men according to the current review probably lies in the origin of individuals that were evaluated. In the review by Parsons et al. 23, only Northern Europe and the United States were represented, but in the current study other countries were included (New Zealand, Spain, and China), among which different associations were reported in men.

These differences in obesity levels between countries have been documented previously. Two systematic reviews of cross-sectional studies conducted in high-income countries showed that obesity was more frequent among poor women 4,8 . This pattern had already been shown in the first European and North American studies published in the 1930s 8 . Meanwhile, among men, the two reviews detected both inverse and direct associations and even lack of association between current socioeconomic status and obesity; importantly, direct associations are less frequent in studies performed in the last 20 years. Meanwhile, in medium and low-income countries, obesity was previously a disease of the socioeconomic elite in both sexes ${ }^{8}$, but since 1980 this association has become predominantly inverse in women, while in men the majority of the studies reported either direct associations or a lack of association 4,5,6. This pattern of nutritional transition is particularly evident in countries that have experienced greater economic growth, affecting women earlier than men 4,6 .

In the current review, the only study held outside of high-income countries found an inverse association with abdominal obesity in women and a direct association in men 26 , thus reflecting a transition pattern. Brazilian studies found a direct association between childhood socioeconomic status and BMI in late adolescence and early adulthood in men 24,25 , and an inverse association in women ${ }^{44}$. Thus, although the results of cross-sectional studies are subject to reverse causality - whereby obesity can determine cur- rent socioeconomic status rather than vice-versa $4,6,8,28,30$-, the transition process described above is consistent with our finding of clearer associations in women than in men for the effects of early poverty. This long-term effect on abdominal obesity appears not to result only from persisting socioeconomic adversities in adulthood, since based on the results of the present review, current socioeconomic status was a partial mediator in the associations.

To understand the associations, it is necessary to contextualize the current results. When the individuals studied in the current review were born, childhood obesity was more frequent among boys and girls from wealthy families, even in high-income countries 8 . This suggests that especially for women, from childhood to adulthood there is a reversal in this association. A similar reversal for girls - but not for boys - was recently reported in a Brazilian cohort 44 . Various studies point to adolescence as the period in which this "socioeconomic reversal of fatness" occurs 7,8,28,45,46,47, apparently as a consequence of biological and cultural factors, although the mechanisms involved are not very clear.

Biologically, women appear to be better adapted than men to survive adverse conditions in early childhood, which is confirmed by higher morbidity and mortality in boys 48,49 . Females have more subcutaneous fat tissue since birth, which favors surviving early adverse conditions 49,50,51. Periods of nutritional restriction in early childhood lead to permanent deficits in the amount of lean mass in adulthood 52, but central adipose tissue appears to be selectively preserved 53. Even if nutrient supply improves over life, central fat distribution is maintained in adulthood, particularly in women, as demonstrated by Ravelli et al. 54 in their study on hunger in the Netherlands during World War II. In adolescence, sex hormones lead to increased fat deposits in women, as an energy reserve to guarantee procreation and breastfeeding 49,50,51, thereby increasing the differences between the sexes. The same hormonal action probably contributes to highlighting the effects of early poverty on central adiposity in women.

Behavioral characteristics of poor women in adolescence may also contribute to the socioeconomic reversal of fatness: higher parity 55 and less concern with body image $23,56,57,58$ or weight reduction 23,28,57. Meanwhile, for men, there are similar proportions of individuals from distinct socioeconomic strata who wish to lose or gain weight during adolescence. However, the control measures acquired by adolescents are mainly targeted to gaining body mass (both fat and lean) $23,56,57,58$. 
Other conditions related to childhood socioeconomic status and that can affect obesity patterns in adulthood need to be considered. Various studies point to parental obesity as an important risk factor for the children's obesity, both in childhood and adulthood 3,23,28,59. This could suggest that the observed associations with socioeconomic status are the consequence of parental obesity. None of the studies in the current review included the parents' nutritional status as an adjustment variable. However, the review by Shrewsbury et al. 7 based on cross-sectional studies in children found that in six of the nine articles that evaluated the association between socioeconomic status and childhood obesity and that included parental adiposity as a co-variable, the direction of the associations did not change. This suggests that socioeconomic status is more than a simple marker of parental obesity. At any rate, it is unlikely that genetic factors can explain the differences between men and women according to early childhood socioeconomic status.

Race, which reflects both the inheritance of genetic characteristics and can represent a proxy for socioeconomic conditions, is also associated with obesity 3,23,28,60. The populations evaluated in the current review consisted of quite homogeneous ethnic groups, so they did not allow evaluating this variable's impact on abdominal obesity.

Childhood nutritional habits and patterns of physical activity have also been mentioned as possible explanatory factors for the observed differences. However, these factors have shown conflicting results in different studies 23,28 . These and other factors need to be explored better to define their importance in these associations.

Various limitations should be considered when interpreting the results of the current review. All the studies used the parents' occupation to assess socioeconomic status, with the exception of the Chinese study, which used household assets. However, the categories used to characterize the parents' occupation varied, thus hindering comparison. Even so, in the eight studies in which it was possible to calculate SII $26,32,34,35,36,38,39,43$, the resulting trends were similar to the original estimates. Other important exposure variables were not evaluated, like maternal schooling, which has been identified as a more stable and relevant risk factor than parents' occupation for this type of association 7,8,23,28. Secondly, various studies only present data for WHR, and not separately for waist and hip circumference. These two circumferences contribute in opposite directions to WHR and risk of cardiovascular diseases 12 , and can also be influenced differently by early socioeconomic status. The study from New Zealand did not stratify the results by gender 41 and was thus unable to determine whether the observed inverse association was similar for men and women. Finally, although various studies have reported considerable loss-to-follow-up rates, they do not explain the differences observed between the genders or the consistency of the findings with the results of cross-sectional studies.

Early poverty increases the risk of abdominal obesity in women, but among men the pattern was not as clear. The negative effect of poverty on abdominal obesity in men may become more evident as the nutritional transition progresses. The effects of early poverty on adults' health are shown by studies approaching different outcomes $2,29,52$. According to the current review, abdominal obesity - with its important consequences for morbidity and mortality from degenerative diseases - constitutes an additional risk factor that could be prevented through social policies to reduce the inequalities that exert a permanent influence on growth and development in the early stages of life. 


\section{Resumo}

Condições sócio-econômicas adversas na infância podem exercer efeitos duradouros sobre a saúde de adultos, mas são poucos os estudos longitudinais que avaliaram os efeitos sobre a obesidade abdominal. Estudos transversais em adultos de países de renda média e alta mostram uma associação inversa entre obesidade e posição sócio-econômica atual em mulheres, mas para os homens não se observa um padrão consistente. Entre homens e crianças de ambos os sexos não existe um padrão definido. Foi realizada uma revisão sistemática dos estudos que avaliaram a posição sócio-econômica precoce e o seu efeito na circunferência da cintura, a circunferência do quadril elou na razão cintura-quadril em adultos. Dos 13 trabalhos incluídos (cinco transversais e oito coortes), apenas um foi realizado em um país de renda média, sendo os demais provenientes de países de renda alta. Em todos os estudos, a pobreza na infância esteve associada com maiores niveis de obesidade abdominal em mulheres. Em homens, as associações foram de menor magnitude e não houve consistência entre os estudos em termos da direção do efeito da posição sócio-econômica.

Gordura Abdominal; Obesidade; Pobreza

\section{References}

1. World Health Organization. The global burden of disease: 2004 update. Geneva: World Health Organization; 2008.

2. Jamison DT, Breman JG, Measham AR, Alleyne G, Claeson M, Evans DB, et al. Disease control priorities in developing countries. 2nd Ed. Washington DC: Oxford University Press/World Bank; 2006.

3. Kipping RR, Jago R, Lawlor DA. Obesity in children. Part 1: epidemiology, measurement, risk factors, and screening. BMJ 2008; 337:a1824.

4. McLaren L. Socioeconomic status and obesity. Epidemiol Rev 2007; 29:29-48.

5. Monteiro CA, Conde WL, Popkin BM. The burden of disease from undernutrition and overnutrition in countries undergoing rapid nutrition transition: a view from Brazil. Am J Public Health 2004; 94:433-4.

6. Monteiro CA, Moura EC, Conde WL, Popkin BM Socioeconomic status and obesity in adult populations of developing countries: a review. Bull World Health Organ 2004; 82:940-6.

\section{Contributors}

D. González performed the article search and analysis, organized the content, and wrote the manuscript. A. Nazmi collaborated in the article search and analysis C. G. Victora oriented the literature review and participated in the analyses and drafting of the article.

\section{Acknowledgments}

The authors wish to thank the Wellcome Trust Foundation for the funding to conduct this research. They also thank Drs. Denise Petrucci Gigante and Jonathan Wells for their valuable contributions to the review.
7. Shrewsbury V, Wardle J. Socioeconomic status and adiposity in childhood: a systematic review of cross-sectional studies 1990-2005. Obesity (Silver Spring) 2008; 16:275-84.

8. Sobal J, Stunkard AJ. Socioeconomic status and obesity: a review of the literature. Psychol Bull 1989; 105:260-75.

9. World Health Organization. Obesity: preventing and managing the global epidemic. Report of a WHO consultation. World Health Organ Tech Rep Ser 2000; 894:i-xii, 1-253.

10. de Koning L, Merchant AT, Pogue J, Anand SS. Waist circumference and waist-to-hip ratio as predictors of cardiovascular events: meta-regression analysis of prospective studies. Eur Heart J 2007; 28:850-6.

11. Yusuf S, Hawken S, Ounpuu S, Dans T, Avezum A, Lanas F, et al. Effect of potentially modifiable risk factors associated with myocardial infarction in 52 countries (the INTERHEART study): case-control study. Lancet 2004; 364:937-52. 
12. Canoy D. Distribution of body fat and risk of coronary heart disease in men and women. Curr Opin Cardiol 2008; 23:591-8.

13. Wells JC, Chomtho S, Fewtrell MS. Programming of body composition by early growth and nutrition. Proc Nutr Soc 2007; 66:423-34.

14. Seidell JC, Perusse L, Despres JP, Bouchard C. Waist and hip circumferences have independent and opposite effects on cardiovascular disease risk factors: the Quebec Family Study. Am J Clin Nutr 2001; 74:315-21.

15. Tanner JM. Foetus into man. 2nd Ed. Ware: Castlemead Publications; 1989.

16. Snijder MB, Dekker JM, Visser M, Bouter LM, Stehouwer CD, Kostense PJ, et al. Associations of hip and thigh circumferences independent of waist circumference with the incidence of type 2 diabetes: the Hoorn Study. Am J Clin Nutr 2003; 77:1192-7.

17. Barker DJ. The developmental origins of adult disease. Eur J Epidemiol 2003; 18:733-6.

18. Barker DJ. The developmental origins of adult disease. J Am Coll Nutr 2004; 23(6 Suppl):588S-95S.

19. Ben-Shlomo Y, Kuh D. A life course approach to chronic disease epidemiology: conceptual models, empirical challenges and interdisciplinary perspectives. Int J Epidemiol 2002; 31:285-93.

20. Leon DA. Biological theories, evidence, and epidemiology. Int J Epidemiol 2004; 33:1167-71.

21. Prentice AM, Moore SE. Early programming of adult diseases in resource poor countries. Arch Dis Child 2005; 90:429-32.

22. Black RE, Allen LH, Bhutta ZA, Caulfield LE, de Onis M, Ezzati M, et al. Maternal and child undernutrition: global and regional exposures and health consequences. Lancet 2008; 371:43-60.

23. Parsons TJ, Power C, Logan S, Summerbell CD. Childhood predictors of adult obesity: a systematic review. Int J Obes Relat Metab Disord 1999; 23 Suppl 8:S1-107.

24. Barros AJ, Victora CG, Horta BL, Gonçalves HD, Lima RC, Lynch J. Effects of socioeconomic change from birth to early adulthood on height and overweight. Int J Epidemiol 2006; 35:1233-8.

25. Goldani MZ, Haeffner LS, Agranonik M, Barbieri MA, Bettiol H, Silva AA. Do early life factors influence body mass index in adolescents? Braz J Med Biol Res 2007; 40:1231-6.

26. Schooling CM, Jiang CQ, Lam TH, Zhang WS, Cheng KK, Leung GM. Life-course origins of social inequalities in metabolic risk in the population of a developing country. Am J Epidemiol 2008; 167:419-28.

27. Regidor E. Measures of health inequalities: part 2. J Epidemiol Community Health 2004; 58:900-3.

28. Power C, Parsons T. Nutritional and other influences in childhood as predictors of adult obesity. Proc Nutr Soc 2000; 59:267-72.

29. Williams DR. Race, socioeconomic status, and health. The added effects of racism and discrimination. Ann NY Acad Sci 1999; 896:173-88.

30. Puhl R, Brownell KD. Bias, discrimination, and obesity. Obes Res 2001; 9:788-805.
31. Moher D, Cook DJ, Eastwood S, Olkin I, Rennie D, Stroup DF. Improving the quality of reports of meta-analyses of randomised controlled trials: the QUOROM statement. Quality of Reporting of Meta-analyses. Lancet 1999; 354:1896-900.

32. Kivimaki M, Smith GD, Juonala M, Ferrie JE, Keltikangas-Jarvinen L, Elovainio M, et al. Socioeconomic position in childhood and adult cardiovascular risk factors, vascular structure, and function: cardiovascular risk in young Finns study. Heart 2006; 92:474-80.

33. Kuh D, Hardy R, Chaturvedi N, Wadsworth ME. Birth weight, childhood growth and abdominal obesity in adult life. Int J Obes Relat Metab Disord 2002; 26:40-7.

34. Laitinen J, Pietilainen K, Wadsworth M, Sovio U, Jarvelin MR. Predictors of abdominal obesity among 31-yr-old men and women born in Northern Finland in 1966. Eur J Clin Nutr 2004; 58 : 180-90.

35. Laitinen J, Power C, Jarvelin MR. Family social class, maternal body mass index, childhood body mass index, and age at menarche as predictors of adult obesity. Am J Clin Nutr 2001; 74:287-94.

36. Langenberg C, Hardy R, Kuh D, Brunner E, Wadsworth M. Central and total obesity in middle aged men and women in relation to lifetime socioeconomic status: evidence from a national birth cohort. J Epidemiol Community Health 2003; 57: 816-22.

37. Langenberg C, Kuh D, Wadsworth ME, Brunner E, Hardy R. Social circumstances and education: life course origins of social inequalities in metabolic risk in a prospective national birth cohort. Am J Public Health 2006; 96:2216-21.

38. Regidor E, Banegas JR, Gutierrez-Fisac JL, Dominguez V, Rodriguez-Artalejo F. Socioeconomic position in childhood and cardiovascular risk factors in older Spanish people. Int J Epidemiol 2004; 33:723-30.

39. Regidor E, Gutierrez-Fisac JL, Banegas JR, LopezGarcia E, Rodriguez-Artalejo E. Obesity and socioeconomic position measured at three stages of the life course in the elderly. Eur J Clin Nutr 2004; 58:488-94.

40. Parker L, Lamont DW, Unwin N, Pearce MS, Bennett SM, Dickinson HO, et al. A lifecourse study of risk for hyperinsulinaemia, dyslipidaemia and obesity (the central metabolic syndrome) at age 49-51 years. Diabet Med 2003; 20:406-15.

41. Poulton R, Caspi A, Milne BJ, Thomson WM, Taylor A, Sears MR, et al. Association between children's experience of socioeconomic disadvantage and adult health: a life-course study. Lancet 2002; 360:1640-5.

42. Brunner E, Shipley MJ, Blane D, Smith GD, Marmot MG. When does cardiovascular risk start? Past and present socioeconomic circumstances and risk factors in adulthood. J Epidemiol Community Health 1999; 53:757-64.

43. Lawlor DA, Ebrahim S, Davey Smith G. Socioeconomic position in childhood and adulthood and insulin resistance: cross-sectional survey using data from British women's heart and health study. BMJ 2002; 325:805. 
44. Gigante DP, Minten GC, Horta BL, Barros FC, Victora CG. Nutritional evaluation follow-up of the 1982 birth cohort, Pelotas, Southern Brazil. Rev Saúde Pública 2008; 42 Suppl 2:60-9.

45. Garn SM, Hopkins PJ, Ryan AS. Differential fatness gain of low income boys and girls. Am J Clin Nutr $1981 ; 34: 1465-8$.

46. Braddon FE, Rodgers B, Wadsworth ME, Davies JM. Onset of obesity in a 36 year birth cohort study. Br Med J (Clin Res Ed) 1986; 293:299-303.

47. Power C, Moynihan C. Social class and changes in weight-for-height between childhood and early adulthood. Int J Obes 1988; 12:445-53.

48. Wells JC. Natural selection and sex differences in morbidity and mortality in early life. J Theor Biol 2000; 202:65-76.

49. Wells JC. Sexual dimorphism of body composition. Best Pract Res Clin Endocrinol Metab 2007; 21 415-30.

50. Wilkin TJ, Murphy MJ. The gender insulin hypothesis: why girls are born lighter than boys, and the implications for insulin resistance. Int J Obes 2006; 30:1056-61.

51. Zafon C. Oscillations in total body fat content through life: an evolutionary perspective. Obes Rev 2007; 8:525-30.

52. Victora CG, Adair L, Fall C, Hallal PC, Martorell R, Richter L, et al. Maternal and child undernutrition: consequences for adult health and human capital. Lancet 2008; 371:340-57.
53. Yajnik CS, Fall CH, Coyaji KJ, Hirve SS, Rao S, Barker DJ, et al. Neonatal anthropometry: the thin-fat Indian baby. The Pune Maternal Nutrition Study. Int J Obes Relat Metab Disord 2003; 27: 173-80.

54. Ravelli AC, van Der Meulen JH, Osmond C, Barker DJ, Bleker OP. Obesity at the age of $50 \mathrm{y}$ in men and women exposed to famine prenatally. Am J Clin Nutr 1999; 70:811-6.

55. dos Santos Silva I, Beral V. Socioeconomic differences in reproductive behaviour. IARC Sci Publ 1997; (138):285-308.

56. McCabe MP, Ricciardelli LA. Body image dissatisfaction among males across the lifespan: a review of past literature. J Psychosom Res 2004; 56: 675-85.

57. Moore DC. Body image and eating behavior in adolescents. J Am Coll Nutr 1993; 12:505-10.

58. Wright EJ, Whitehead TL. Perceptions of body size and obesity: a selected review of the literature. J Community Health 1987; 12:117-29.

59. Tequeanes AL, Gigante DP, Assunção MC, Chica DA, Horta BL. Maternal anthropometry is associated with the body mass index and waist:height ratio of offspring at 23 years of age. J Nutr 2009; 139:750-4.

60. Wagner DR, Heyward VH. Measures of body composition in blacks and whites: a comparative review. Am J Clin Nutr 2000; 71:1392-402.

Submitted on 30/Mar/2009

Final version resubmitted on 11/Sep/2009

Approved on 23/Sep/2009 